\title{
Calculation of electron scattering cross-section using different theoretical methods
}

\author{
Xiazhi $\mathrm{Li}^{1, *}$, Jinhui Zhu ${ }^{1, * *}$, Yinghong Zuo ${ }^{1}$, and $Y a \mathrm{Li}^{1,2}$ \\ ${ }^{1}$ Northwest Institute of Nuclear Technology, 410079, Xi' an, China \\ ${ }^{2}$ Department of Engineering Physics, Tsinghua University, 100084, Beijing, China
}

\begin{abstract}
Aiming at the simulation of the fine process of the electron transportation in gamma detectors, we calculate electron differential scattering cross-section (DCS) of several typical materials including Fe, ethylene and polyethylene. Based on two different calculation methods, which are partial wave methods based on Dirac equation and R-matrix theory, we find differences of the cross section at low energy region. The result indicates that both partial wave method and R-matrix theory associated with independent atom method (IAM) are not suitable for low energy electron impacting on strong coupled molecule, for example, electron-ethylene. For high energy electron interacting with atom and molecule, the result shows no critical difference because the kinetic energy of the incident electron is severely higher than the electron bound energy in molecule or the excitation energy of a certain atom.
\end{abstract}

\section{Introduction}

Plastic scintillator, compared with the inorganic scintillator, has characteristics including low light yield, short rising time and decay time, etc. Based on these differences, plastic scintillators are usually used in the fast time resolution measurement [1]. Both time resolution and energy response are decided by electron energy deposition [2]. Thus, the research of electron transportation is significant since the appearance of scintillators. Here we focus on the low energy region as two long standing problems, energy response nonlinearity and time resolution, lies in this region. The fundamental task is to calculate low energy electron cross section.

As is well known, partial wave method is one of the basic methods in low energy electron cross section calculation. Mott [3] demonstrated its relativistic edition and processed some electron-atom scattering problems. R-matrix method is well developed by Burke [4] and intensely used in the calculation of opacity [5]. For small molecules, Schwinger multi-channel method(SMC) [6] can give some satisfying outcomes in low energy region [7]. Independent atom method (IAM) [8] is also frequently used to calculate electron-molecule cross section for its short time consuming compared to SMC.

In this paper, we have demonstrated the total cross section of the electron on specific atoms including $\mathrm{C}, \mathrm{H}$ and Fe. More than that, total cross sections of ethylene and polyethylene molecules are also calculated using IAM method combing screen correlation additivity rule (SCAR). To our knowledge, there is no available data on the electron cross section of polyethylene. Thus, the present result can be only considered as a theoretical exploration.

\section{Theory}

In Mott's [3] theory about partial wave method, the scattering wave can be expanded into an outgoing spherical wave plus a plane wave. The differential cross section (DCS) per solid angle is given by [3]:

$$
d \sigma / d \Omega=|f(\theta)|^{2}+|g(\theta)|^{2}
$$

in which $f(\theta)$ is the direct scattering amplitude and $g(\theta)$ is spin-orbital scattering amplitude. The scattering amplitude can be expanded into spherical harmonics, namely

$$
\left\{\begin{array}{c}
f(\theta)=\frac{1}{2 i k} \sum_{l=0}^{\infty} A_{l} P_{l}(\cos \theta) \\
A_{l}=(l+1)\left[\exp \left(2 i \delta_{k=-l-1}\right)-1\right]+l\left[\exp \left(2 i \delta_{k=l}\right)-1\right]
\end{array}\right.
$$

and

$$
g(\theta)=\frac{1}{2 i k} \sum_{l=0}^{\infty}\left[\exp \left(2 i \delta_{l}\right)-\exp \left(2 i \delta_{-l-1}\right)\right] P_{l}^{1}(\cos \theta)
$$

$k$ is the wave number of projectile. $\delta_{k=l}$ is the phase shifting for partial wave $l . \theta$ is the scattering angle relative to the incident electron momentum direction. $P_{l}(\cos \theta)$ and $P_{l}^{1}(\cos \theta)$ are Legendre polynomials and associated Legendre polynomials respectively. Once we have obtained DCS of a certain target, the total cross section is derived by integrating DCS in the whole solid angle,

$$
\sigma=\int \frac{d \sigma}{d \Omega} d \Omega=\int \frac{d \sigma}{d \Omega} 2 \pi \sin \theta d \theta .
$$

\footnotetext{
*e-mail: 1xznudt2015@hotmail.com

**e-mail: zhujinhui@nint.ac.cn
} 
Partial wave method can be a good approximation when the incident electron energy is not that low. For the lower energy, the DCS can be well described by R-matrix theory. There is a detailed edition of this theory by Burke [4].

So far, we have discussed the scattering process on an atom target. For detectors, the components of scintillator, however, are molecules. Hence, the calculation of electronmolecule cross section is of more interest. IAM is one of the approximate methods to calculate this process. The first edition of IAM treated the electron-molecule scattering as series of independent scatterings between atoms in one molecule and these internal scatterings are irrelevant. The cross section of electron-molecule is a direct sum of electron-atom cross sections, known as the usual additivity rules (AR) [8]

$$
\sigma_{\text {total }}=\sum_{\text {atoms }} \sigma_{i}
$$

Although IAM works well for moderate to large energy, it is not expected to be a good approximation for low energy as the cross section in this energy region is comparable to the area determined by the bound length in a given molecule. This indicates that the incident electron is very likely to interact with more than one atom at the same time. While in IAM-AR condition, the electron is still scattered between atoms one by one resulting that the contribution of a certain atom to the total cross section may be heavily overestimated. To weaken this effect, it is naturally to minimize the screen (overlap) effect between atoms. Independent atom method combining screen correlation additivity rules (IAM-SCAR) is used to evaluate the overlapping. In this method, the fixed electron-molecule total cross section can be expressed by replacing the AR cross section with modified SCAR cross section [9]

$$
\sigma_{\text {total }}=\sum_{i} s_{i} \sigma_{i}
$$

in which the sum should extend to all the atoms in the given molecule and $s_{i}$ is the screening coefficient $\left(0 \leq s_{i} \leq\right.$ 1). Total cross section $\sigma_{i}$ and the respective position of each atom are the only requirements in the calculation of $s_{i}$. Here we will not give the detailed derivation of screening coefficients but the explicit expression[9]:

$$
\begin{gathered}
\varepsilon_{i}^{(k)}=\frac{\frac{N-k+1}{N-1} \sum_{j(j \neq i)} \sigma_{j} \varepsilon_{i}^{(k-1)}}{\alpha_{i j}}(k=2,3, \ldots, N) \\
s_{i}=1-\frac{\varepsilon_{i}^{(2)}}{2 !}+\frac{\varepsilon_{i}^{(3)}}{3 !}+\cdots \pm \frac{\varepsilon_{i}^{(N)}}{N !}=\sum_{n=1}^{N} \frac{(-1)^{n-1}}{n !} \varepsilon_{i}^{(n)}
\end{gathered}
$$

in which $N$ stands for the number of atoms in the molecule, the index $j$ should run over all the atoms except the $i$ th one, $\alpha_{i j}=\max \left(4 \pi r_{i j}^{2}, \sigma_{i}, \sigma_{j}\right), \sigma_{i}$ is the cross section of the $i$ th atom and $r_{i j}$ is the distance between the $i$ th and $j$ th atoms. As there is no screening effect at all for an independent atom, we naturally have $\varepsilon_{m}^{(1)}=1$. The number $k$ starts from 2 and ends at the final atom, so the term $\varepsilon_{i}^{(2)}$ stands for the diatoms. Usually the molecule cross section using SCAR procedure is significantly smaller than AR procedure. The application of SCAR to small molecule is very convenient and the time consuming is short enough. However, the complication of calculation rises severely when applying this method to macromolecule. The reason is quite simple that there are too many atoms in a given molecule. To simplify this problem, one can change the calculation from individual atom to each atom cluster. For example, the components of protein are amino acids. Once the cross section of each amino acid is calculated, it can be treated as a micro-atom to the protein and the cross section of the macromolecule can be derived from these small blocks. This method is the so-called SCAR-M mothed. Similar to SCAR method, the cross section of the macromolecule is a weighted sum of each small molecule cross section, namely

$$
\sigma^{M M}=\sum_{i} s_{i}^{M} \sigma_{i}^{M}
$$

where $\sigma^{M M}$ stands for the cross section of the macro molecule and $\sigma_{i}^{M}$ is the cross section of the $i$ th small molecule. As mentioned above, the only information needed in SCAR total cross section is atom total cross section and position. The requisite inputs for SCAR-M are total cross section and position of each small molecule. Total cross section of small molecule can be calculated by SCAR on each small block. The position of each small molecule is weighted by its cross section

$$
\bar{r}^{M}=\sum_{\text {atomsin } M} r_{j}^{\text {atom }} \sigma_{j}^{\text {atom }} / \sum_{\text {atomsin } M} \sigma_{j}^{\text {atom }} .
$$

The definition of $\bar{r}^{M}$ is quite like 'center of gravity' but it is decided by atom cross section instead of the atom mass. It should be noted that SCAR-M is a further approximation to SCAR. The internal orientation is neglected. Fortunately, this approximation does not have a significant effect on total cross section.

\section{Numerical calculation and discussion}

For a given scintillator, it is made up by scintillator media and a shell while the following photon-to-electron and digital system are not discussed here. To simplify the problem, we assume that shell is $\mathrm{Fe}$ and the scintillator is polyethylene $(\mathrm{C}, \mathrm{H})$. It is noted that the real situation is far from the hypothetical parameters and the calculation is a theoretical demonstration of such system.

\subsection{Cross section of atoms}

In this paper the cross section of molecule is given through IAM procedure which is based on atom cross section and position. Thus the basic calculation should be the cross section of neutral atoms in the whole system. Fig 1 is the elastic cross section of Fe-56, C-12 and $\mathrm{H}-1$ in which the $\mathrm{R}$-matrix data are referred to Cullen's [10] work. It is clear that the cross section decreases as the energy increases in the given region (the cross section does not always obey such rules). Considering the electron as a matter wave, as the energy rises, the size of the incident electron wavelet is being smaller. This results in the reduction of the area that the electron can directly influence. Fig 2 is the DCS of Fe 


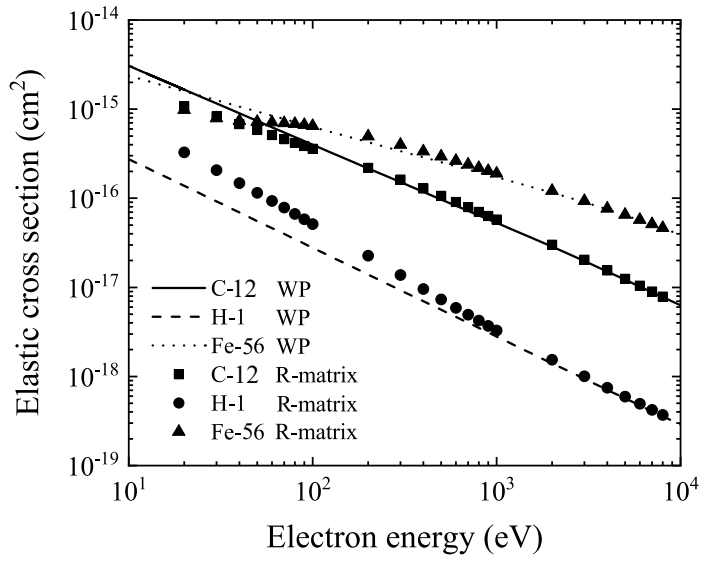

Figure 1. Cross section of $\mathrm{C}, \mathrm{H}, \mathrm{Fe}$ with electron energy above $10 \mathrm{eV}$. WP stands for wave partial method

at some energy points. As we expand the scattering electron wave by Legendre polynomials, the DCS in both low and high energy regions are symmetric about the incident direction. For the high energy electron, it can penetrate the target barrier deeper and thus, the phase shift $\delta_{\kappa=l}$ decreases slowly with $l$. This leads to a sharply peak at $\theta=0$. For the low energy electron, the target barrier prevents it from approaching the sacttering center. High order waves are not affected by the barrier and the phase shift $\delta_{l}$ decreases rapidly with order $l$. Thus, the DCS of low energy electrons is determined by a few low order phase shifts. Ultimately, this results in a clear space variation. This explanation can be only used in the elastic collision in principle. For convenience and accuracy, the next calculations are based on data acquired by R-matrix theory. Besides, we use total cross section instead of elastic cross section to demonstrate cross section calculation of molecule and macromolecule.

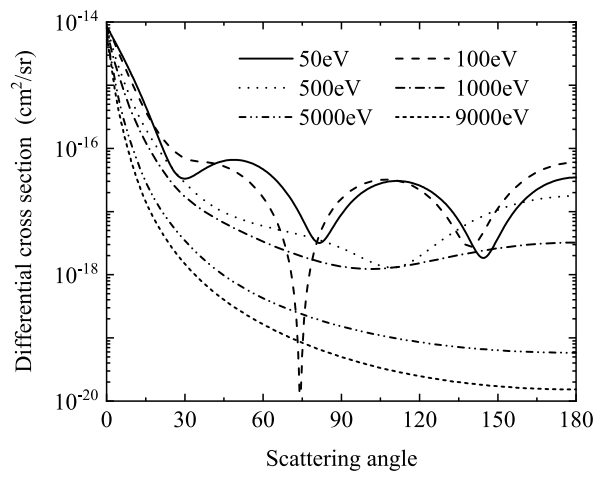

Figure 2. DCS of total cross section of atomic $\mathrm{Fe}$ at different incident electron energy

\subsection{Cross section of ethylene molecule}

IAM-SCAR method is used to the cross section calculation of the ethylene molecule. As we have mentioned above, the requisite information for SCAR total cross section is atom cross section and position. In section 3.1, we have given the cross section of the component atoms, namely carbon and hydrogen. Data for the position of the internal atoms can be downloaded from the chemistry book of NIST [11]. As the ethylene molecule is planar, all the atoms are in the same plane. The central symmetry promises that the screening coefficient is the same for each carbon and hydrogen. Based on this symmetry, we only need to calculate the screening coefficient of one carbon and hydrogen.

Fig 3 shows the total cross section of ethylene vs electron

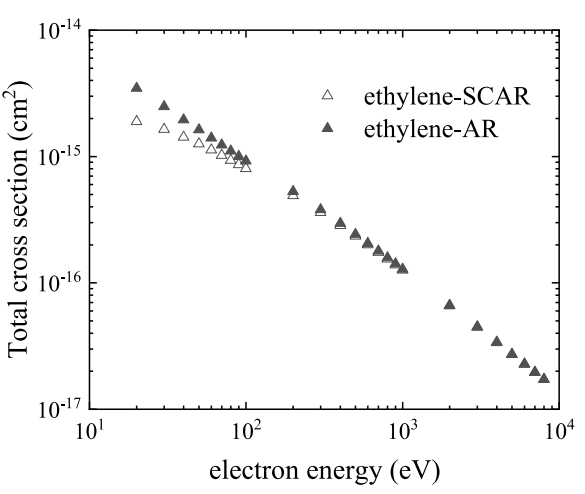

Figure 3. Total cross section of atomic Fe at different incident electron energy

energy using different additivity rules. The figure shows a clear discrepancy at low energy. Cross section using SCAR method can be $50 \%$ lower than the AR method. The result indicates a strong screen effect at low incident energy as we have explained before. For energy larger than $1 \mathrm{keV}$, the difference between SCAR and AR method is not apparent. In this energy region, the cross section is much smaller than the bound length in the given molecule. In section 2 we have pointed out that in this condition, the incident electron interacts with internal atoms independently.

\subsection{Cross section for polyethylene}

The cross section calculation needs a static molecule model in which the components of the given molecule should not vary. However, the atom number in a polyethylene molecule is not constant. Besides, the geometries of polyethylene molecules are variant. It is impossible to calculate actual types of geometries and then sum them up. Here we simply assume that the atoms in polyethylene molecule are in the best position without disturbance from other molecules. This assumption results in an alkane hydrocarbon like macromolecule in which all the carbons are in the same plane. If we visualize the whole molecule, it should be a straight line. For polyethylene, the number of carbon atoms is far more than 1 and we treat it as an kind of alkane hydrocarbon with $n \gg 1$. Based on this, we split the polyethylene into same blocks by cluster $-\mathrm{CH}_{2}-$. Then, the requisite information for SCAR-M 


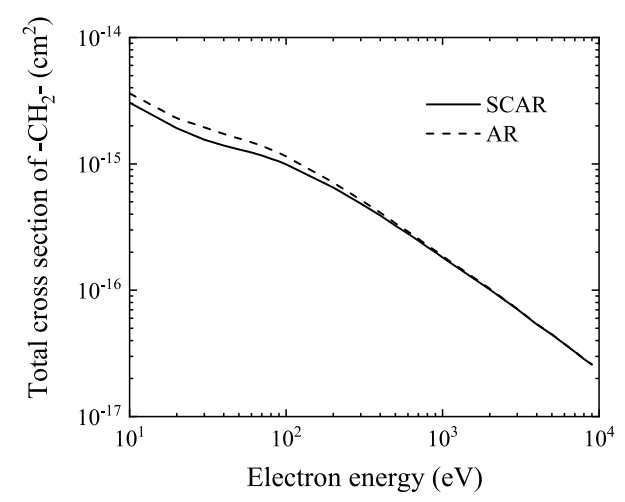

Figure 4. Total cross section of $\mathrm{CH}_{2}$ at different incident electron energy

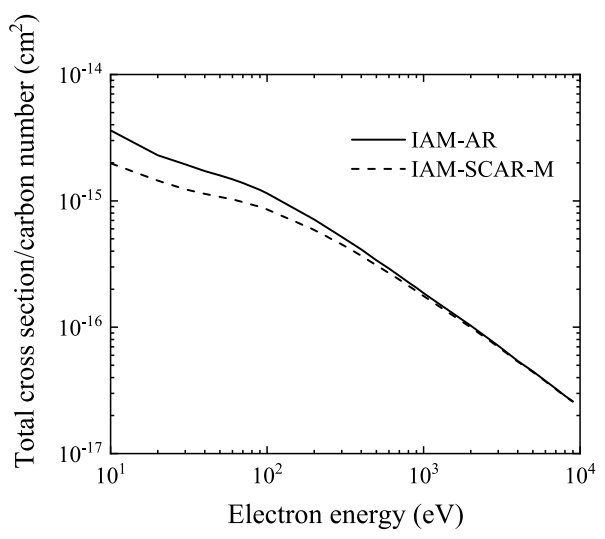

Figure 5. Total cross section of polyethylene at different incident electron energy

method is the location and cross section of cluster $-\mathrm{CH}_{2}-$. To further simplify the question, we assume that each cluster in polyethylene is just like the $\mathrm{C} 1$ and $\mathrm{C} 2$ in nonane molecule in which the whole molecule is symmetric to any carbon atom. The cross section of $-\mathrm{CH}_{2}-$ can be deduced by SCAR method and the result is shown in Fig 4. The result is similar to Fig 3 as they are derived by the same SCAR method. Choosing the cross section of $-\mathrm{CH}_{2}-$ as $\sigma_{i}^{M}$ to SCAR-M method, we need only the position of each equivalent molecule. As we admit that the macromolecule has an infinite length, the structure of such molecule should be strictly symmetric. Thus, the inner atoms in nonane can be a good approximation to polyethylene. Applying the center of gravity method to cluster $-\mathrm{CH}_{2}-$, the distance between neighboring clusters can be calculated. As the atom number in the chain is infinite, they are all the same in SCAR-M method. The homosexuality promises the uniformity in coefficients $s_{i}$ and its each term $\varepsilon_{i}^{(k)}$. We only need to give a whole expansion of one small molecule then the other expansions are the same to it. In real calculation, a cut-off is induced to avoid meaningless summation. Here we simply think there are 1000 small molecule in a polyethylene. Fig 5 shows the cross section of polyethylene. One can see the clear discrepancy at low energy using different additivity rules.

\section{Conclusion}

We have calculated the total cross section of the polyethylene and its monomer ethylene by SCAR-M and SCAR methods respectively. Results show that in low energy region, the screening effect significantly decreases the cross section while in high energy region, this effect can be neglected and the electron can be treated as scattering between different atoms independently. It indicates that the traditional calculation method complying with direct additivity rules may underestimate the range of low energy electrons. Cross section additivity rules should be carefully selected under different energy regions.

\section{References}

[1] Zaitseva N, et al, Nuclear Instruments \& Methods in Physics Research 668, 88-93 (2012)

[2] Williamson J F, Dempsey J F, Kirov A S, Physics in Medicine \& Biology 44, 857-871 (1999)

[3] Mott N F, Proceedings of the Royal Society A Mathematical Physical \& Engineering Sciences 127, 658-665 (1930)

[4] Burke P G, Noble C J, Scott P, Proceedings of the Royal Society of London 410, 289-310 (1987)

[5] Nahar S N, Pradhan A K, Astronomy \& Astrophysics 279, 298-309 (2004)

[6] Lima M A, Brescansin L M, Da S A, et al, Physical Review A 41, 327-332 (1990)

[7] Gibson T L, Mckoy V, Lima M A P, et al, Physical Review A 30, 3005-3011 (1984)

[8] Blanco F, Garcia G, Physics Letters A 317, 458-462 (2003)

[9] Blanco F, Rosado J, Illana A. Garcíab G, Physics Letters A 374, 420-424 (2010)

[10] Cullen D E, A Survey of Electron Cross Section Data for use in EPICS2017, IAEA-NDS-0226 (2018)

[11] LINSTROM, Peter J, William G, Journal of Chemical \& Engineering Data 46, 1059-1063 (2003) 